\title{
RELATIVISTIC CALCULATIONS OF EXCITATION AND IONIZATION OF HIGHLY CHARGED IONS BY ELECTRON IMPACT
}

\author{
Final Technical Report for
}

Grant DE-FG02-90ER54104

\author{
Douglas H. Sampson \\ The Pennsylvania State University \\ University Park, PA 16802
}

\begin{abstract}
NOTICE
This report was prepared as an account of work sponsored by the United States Government. Neither the United States Department of Energy, nor any of their employees, nor any of their contractors, subcontractors, or their employees, makes any warranty, expressed cr implied, or assumes any legal liability or responsibility for the accuracy, completeness, or usefulness of any information, apparatus, product or process disclosed, or represents that its use would not infringe privately owned rights.
\end{abstract}

April 15, 1992

Prepared for

THE UNITED STATES DEPARTMENT OF ENERGY

UNDER Grant No. DE-FG02-90ER54104 
TABLE OF CONTENTS

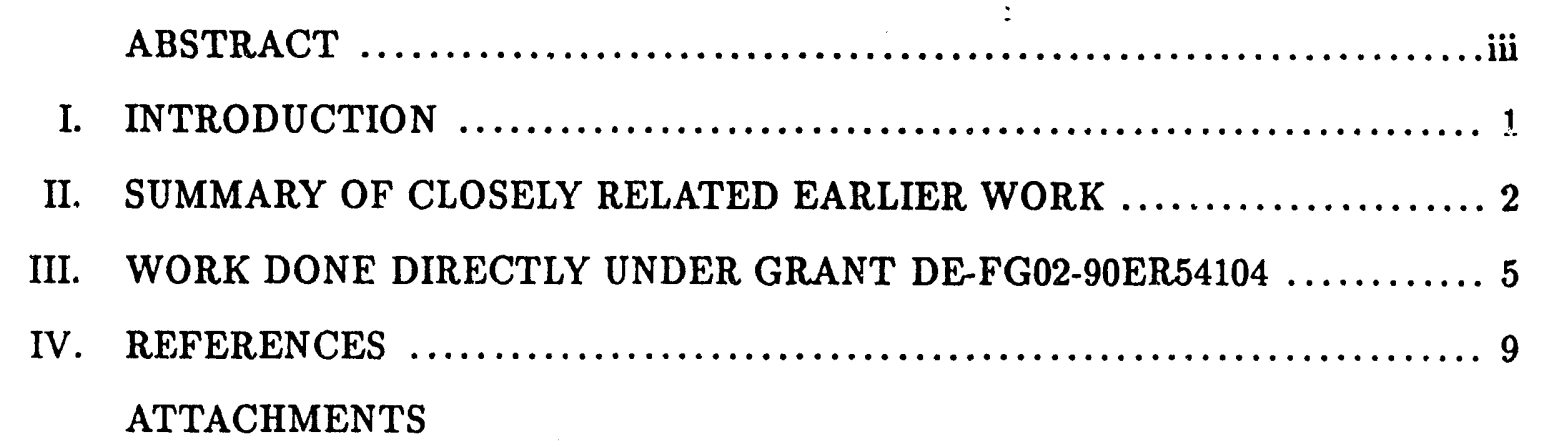

D.H. Sampson, H.L. Zhang and C.J. Fontes, "Relativistic Distorted Wave Collision Strengths and Oscillator Strengths for F-Like Ions with $22 \leq Z \leq 92^{\text {n }}$, Atom. Data Nucl. Data Tables, 48, 25-90 (1991).

H.L. Zhang, D.H. Sampson and C.J. Fontes, "Relativistic Distorted Wave Collision Strengths and Oscillator Strengths for the $33 \mathrm{Ni}$-like Ions with $60 \leq Z \leq 92$ ", Atom. Data Nucl. Data Tables., $\underline{48}$, 91-163 (1991).

H.L. Zhang and D.H. Sampson, "Relativistic Distorted Wave Collision Strengths and Oscillator Strengths for the $45 \Delta n=0$ transitions with $n=2$ in the 85 Be-Like Ions with $8 \leq Z \leq 92^{\prime \prime}$, accepted by Atom. Data Nucl. Data Tables.

D.H. Sampson and H.L. Zhang, "Rapid Relativistic Ionization Approach Based on the Factorization Method", Phys. Rev. A $\underline{45}$, 1657-1669 (1992).

M.K. Inal, H.L. Zhang and D.H. Sampson, "Circular Polarization of the FeXXV X-ray Lines Following Collisional Excitation by Longitudinally Polarized Electrons", accepted by Phys. Rev. A.

D.H. Sampson and H.L. Zhang, "On Use of the Van Regemorter Formula for Collision Strengths or Cross Sections”, Phys. Rev. A. $\underline{45}, 1556-1561$ (1992). 


\begin{abstract}
Our rapid relativistic atomic structure program and relativistic distorted wave programs for excitation and ionization of highly charged ions were further improved with respect to both accuracy and efficiency. First the generalized Breit interaction and other QED corrections were added to the atomic structure program and the speed of the distorted wave excitation program was increased by over an order of magnitude over what it was when our initial large scale relativistic calculations of excitation of Ne-like ions were made. The improved programs were then used to calculate collision strengths for 330 transitions in F-like ions with $22 \leq Z \leq 92$ and 248 transitions in Ni-like ions with $60 \leq Z \leq 92$. In order to further improve the accuracy and extend the range of validity of our calculations, we expanded our relativistic collision program to include an option to use atomic structure data by the well-known multi-configuration Dirac-Fock (MCDF) program of Grant and coworkers. This was used in calculating collision strengths for the $45 \Delta n=0$ transition» with $n=2$ in Be-like ions with $8 \leq Z \leq 92$. This relativistic collision strength program was also extended to include an option to include the generalized Breit interaction in the scattering matrix elements and the importance of this for H-like, He-like and Li-like ions with $Z=26,54$ and 92 was studied. The factorization method was applied to ionization and it was found that regardless of the complexity of the ion the ionization cross sections could be written as a sum of the products of a readily calculated coefficient that depends only on ion properties and a hydrogen-like cross section. The latter has the same form as for hydrogenic ions, but with the radial functions calculated using the appropriate potential for the actual ion. In addition, work was done on excitation and ionization by directive and, in some cases.spin polarized electrons, which is of interest for some EBIT experiments and the study of solar flares. We also used our extensive collision strength results to test the reliability of the well-known and widely used Van Regemorter approximation.
\end{abstract}




\section{INTRODUCTION}

This is the final technical report on the work done under Grant DE-FG02-90ER54104. This was a continuation of work previously supported by the Division of Applied Plasma Physics, Office of Fusion Energy, U.S. Department of Energy first under Contract EY-76-S-02-2897, later called Contract DE-AC02-76ET53056, then under Grant DE-FG02-85ER53208, and finally under Grant DE-FG02-90ER54104. Also in 1985 we began receiving partial support for work in this area from the Lawrence Livermore National Laboratory (LLNL) under Subcontract 6181405 and in 1988 from the Ultrashort Wavelength Laser Program, Innovative Science and Technology Office, Strategic Defense Initiative Organization (SDIO) under Contract N00014-88-K-2021. However, both of these two contracts expired the late summer of 1990. Since then with their own budgets reduced, LLNL has only been able to provide us with an increment of $\$ 20,000$, while SDIO has only provided us with $\$ 15,000$. It is hoped that both of these will be able to provide at least a small amount of support for this work in the future.

The purpose of the work reported here is to calculate the atomic data needed for application to the study and understanding of very high temperature plasmas such as occur in fusion energy research, astrophysical research and research to develop X-ray lasers. In particular, we have been calculating the atomic data needed to model and diagnose such plasmas. For fusion energy research this includes data needed to determine energy losses due to radiation by impurity ions. Our main emphasis has been on calculations of cross sections for excitation and ionization of highly charged ions by electron impact. This was done partly because these processes tend to be the most lengti:y to calculate and there appeared to be the greatest deficiency in atomic data of this kind. However, we have also been calculating relativistic radiative oscillator strengths and we have recently expanded our programs to give a rapid relativistic autoionization program.

In the next section we review our earlier work in this area, then in Section III we discuss the more recent work done during the period of Grant DE-FG02-90ER54104, which was September 15, 1990 - December 14, 1991. 


\section{SUMMARY OF CLOSELY RELATED EARLIER, WORK}

Since an immense amount of atomic data is required for high temperature plasma applications, a very rapid method of calculation is needed. In Ref. 1-46 we developed and applied a very rapid approach using hydrogenic basis functions and screening constants with both the electron-electron Coulomb interaction and relativistic interactions included by perturbation theory.

More recently, in order to improve the accuracy and extend the range of validity of our results, we have in Ref. 47 and 48 developed a new, very rapid fully relativistic approach based on the Dirac equation with the same relativistic Hartree-Fock-Slater or so called Dirac-Fock-Slater (DFS) central potential used for calculating the orbitals for all electrons, bound and free. Hence, all orbitals are automatically orthogonal and both the dominant part of the electron-electron interaction and the dominant relativistic interactions are included in the zeroth order Hamiltonian rather than being treated perturbatively.

This fully relativistic approach, like the earlier approach, is well suited for treating a given class of transitions in all ions within a large portion of an isoelectronic sequence simultaneously. In Ref. 49-52 we have applied this approach to large scale production of atomic data. In Ref. 49 it was used to obtain collision strengths, or equivalently cross sections, for the 88 transitions from the ground level to the excited levels vith $n=3$ and 4 in the 71 neon-like ions with nuclear charge number $Z$ in the range $22 \leq Z \leq 92$. In Ref. 50 similar calculations were made for the 66 transitions among the $2 s_{1 / 2}, 2 p_{1 / 2}$ and $2 p_{3 / 2}$ levels and from them to all the $n l j$ levels with $n=3$, 4 and 5 in the $85 \mathrm{Li}$-like ions with $\varepsilon \leq Z \leq 92$. In Ref. 51 similar calculations were made for the 90 transitions among the $3 s_{1 / 2}, 3 p_{1 / 2}, 3 p_{3 / 2}, 3 d_{3 / 2}$ and $3 d_{5 / 2}$ levels and from them to all the $n l j$ levels with $\mathrm{n}=4$ and 5 in the 71 -Na-like ions with $22 \leq Z \leq 92$. In Ref. 52 similar calculations were made for the 21 transitions among the $4 s_{1 / 2}, 4 p_{1 / 2}, 4 p_{3 / 2}, 4 d_{3 / 2}, 4 d_{5 / 2}, 4 f_{5 / 2}$ and $4 f_{7 / 2}$ levels and the 63 transitions from them to the excited $n l j$ levels with $n=5$ in the $33 \mathrm{Cu}$-like ions with $60 \leq Z \leq 90$.

In order to carry out large scale calculations as in Ref. 49 - 52 efficiently we use several procedures all of which have been well tested and introduce maximum errors at about the $1 \%$ level. One of these procedures is that in order to reduce the number of radial functions and radial scattering integrals we calculate them for a fixed set of final, or scattered electron energies (usually 
six) starting near zero and going out to sufficiently high values to allow accurate determination of collision rates. For each of these we compute the radial integrals for three impact electron energies spanning the range of transition energies for the class of transitions being considered. Then we interpolate on these results to obtain values for the exact transition energy in each case. It should be mentioned that, although we generally calculate collision strengths for a fixeả set of final electron energies in our large scale calculations, our computer programs have the option to calculate collision strengths for a set of impact electron energies, which are sometimes preferred by some scientists. If the factorization method of Bar-Shalom et al ${ }^{53}$ is used, the interpolation is done on the $Q^{\lambda}$. In general we do use the factorization method ${ }^{53}$ whenever we do large scale calculations for a big portion of an isoelectronic sequence, as in Ref. $49-52$. Then the collision strength $\Omega$ has the form

$$
\Omega=8 \sum_{\substack{j_{a}, j_{a}^{\prime} \\ j_{a}, j_{a 1}^{\prime}}} \sum_{\lambda} B^{\lambda}\left(j_{a} j_{a}^{\prime}, j_{a 1} j_{a 1}^{\prime}\right) Q^{\lambda}\left(j_{a} j_{a}^{\prime}, j_{a 1} j_{a 1}^{\prime}\right)
$$

Here $j_{a 1}$ and $j_{a}$ represent orbitals in the initial target ion wave function, and $j_{a 1}^{\prime}$ and $j_{a}^{\prime}$ represent orbitals in the final target ion wave function. A convenient feature of $\mathrm{Eq}(1)$ is that $B^{\lambda}$ is a function only of target ion quantum numbers and mixing coefficients plus $\lambda$, while the $Q^{\lambda}$ contains the radial contribution and depends only on $\lambda$ and the bound and free orbitals with the summations over the free-electron momenta performed within it. Since the $Q^{\lambda}$ vary smoothly with $Z$, in treating a given class of transitions for a large portion of an isoelectronic sequence, one needs to make detailed calculations of the $Q^{\lambda}$ for only a few values of $Z$ (typically one in ten) and then these can be fitted to a power series in $Z$ that generally gives accurate results to within a percent for the complete range of $Z$ being considered. For example, in Ref. 49 detailed calculations were made for the $Z$ values $22,30,42,56,74$ and 92 . Then each $Q^{\lambda}$ was fit to the form

$$
Z^{2} Q^{\lambda}=a_{1}^{\lambda} Z^{3}+a_{2}^{\lambda} Z^{2}+a_{3}^{\lambda} Z+a_{4}^{\lambda}+a_{5}^{\lambda} Z^{-1}+a_{6}^{\lambda} Z^{-2}
$$

Since usually the $Q^{\lambda}$ calculation is the lengthy part, this procedure typically leads to a factor of 10 reduction in computing time.

A quasirelativistic option is also included in our programs. This corresponds to dropping the sniall component of the radial functions and normalizing the large component as though it were 
the complete radial function. In addition, for the free electrons one uses the average (over $j$ ) value $\kappa=-1$ in the effective potential determining the free radial functions. They then become independent of $j$. This is a good approximation because one sums over $j$ values for the free electrons in calculating the collision strength. Although as discussed in Ref. 2 the quasirelativistic results are quite accurate for most $Z$ values, it only saves about a factor of 2.5 in computing time and it was not used in the large scale calculations of Ref. $49-52$.

Our collision program was also expanded to give a rapid program for relativistic distorted wave electron impact ionization cross sections in Ref. 54 . This gives generally good agreement with other recent relativistic ionization work done in Ref. $55-59$. The program of Ref. 54 calculates cross sections $Q_{H}^{p s}\left(n_{a} l_{a} j_{a}\right)$ with the form of hydrogenic cross sections except that the orbitals are calculated using the Hartree-Fock-Slater potential for the target ion. Hence, it can obviously be applied to ionization of the valence electron in Li-like, $\mathrm{Na}$-like and $\mathrm{Cu}$-like ions. However, as shown in the Appendix of Ref. 42, such a program has much wider applications. In fact, we have very recently shown that in general ionization cross sections can be expressed in terms of the $Q_{H}^{p s}$, see Eq. (4) below; as long as mixing is confined to all states within a complex, which is generally adequate for highly charged ions.

It is also convenient to express the $Q_{H}^{p s}$ in terms of a reduced cross section $Q_{R}$ by factoring out a $\pi a_{o}^{2} / I(\mathrm{Ryd})^{2}$ factor, where $I$ is the ionization energy. Then, as shown in Ref. 54, the relativistic distorted wave values for the $Q_{R}$ are close to the non-relativistic Coulomb-Born-Exchange values of Ref. 17 for hydrogenic ions $Q_{R}^{H}$ except for high $Z$ and/or energies. Since the $Q_{R}^{H}$ have been fitted to simple functions of the electron energy in threshold units that are readily integrated over a Maxwellian electron distribution function to obtain ionization rates, one would expect that accurate fits of the relativistic results for the reduced cross section could be made, as well, probably using the same functional form given by $\mathrm{Eq}(6)$ of Ref. 17, but allowing the coefficients to be slowly varying functions of an effective $Z$ or of $Z$ and $N$, where $N$ is the number of bound electrons per ion. This would be very convenient for applications to plasma modeling.

In addition to the type of work just described, which was the main thrust of our effort, we have in Ref. 60 made a version of our relativistic collision strength program that gives cross sections for excitation to specific magnetic sublevels of the target ion by directive electrons. This was done at 
the request of Livermore for use in comparing with and interpreting the electron beam ion trap (EBIT) experiments being done there. They have made measurements for $\mathrm{Ba}^{46+}$, as well as other ions ${ }^{61-63}$ and expect to go to considerably higher values of $Z$ in future work. ${ }^{64}$ The results by this program are of similar interest for EBIS experiments and results for that purpose have been sent to C. Bhalla at Kansas State at his request. ${ }^{65}$ They are also of interest for solar applications.

\section{WORK DONE DIRECTLY UNDER GRANT DE-FG02-90ER54104}

Now we consider the work done specifically under Grant DE-FG02-90ER54104. First we discuss improvements made in the relativistic atomic structure code. As noted in Sec. III A of Ref. 47, the accuracy of our approach appeared to warrant the inclusion of the generalized Breit interaction and other QED corrections in our atomic structure program. These can be significant for very high $Z$, especially in complex ions such as fluorine-like ions. Hence, before carrying out the very lengthy calculations of the collision strengths for all 330 transitions among the $n=2$ levels and between the $n=2$ and $n=3$ levels in the $71 \mathrm{~F}$-like ions with $22 \leq Z \leq 92$ reported in Ref. 66 (reprint attached), we included these modifications or corrections to our relativistic atomic structure program. In addition, we replaced the point nuclear charge in the DFS potential used in calculating the bound and free radial functions with a finite nuclear charge having the Fermi distribution. ${ }^{67}$ These modifications were also included in the calculations of the collision strengths for all 248 possible transitions from the ground level to the $n=4$ and $n=5$ levels in the $33 \mathrm{Ni}$-like ions with $60 \leq Z \leq 92$ recently given in Ref. 68 (reprint attached).

It should also be mentioned that the speed and efficiency of our relativistic codes have been improved considerably. For example, the speed of our relativistic distorted wave program for excitation has been increased by over an order of magnitude since our first large scale calculations given in Ref. 49 for Ne-like ions were made. As an example of the present speed of the codes we note that the calculations for 6 scaled scattered electron energies for each of 330 transitions in each of 71 F-like ions with $22 \leq Z \leq 92$ made in Ref. 66 required less than 50 minutes CPU time on a Cray XMP. This amounts to about 0.02 second per collision strength data point.

With regard to accuracy, as discussed in Ref. $47-52$ and in Ref. 66 and 68, our relativistic 
approach appears to be accurate to within a few percent for $Z \gtrsim 2 N$, where $N$ is the number of bound electrons per ion. In order to further increase the accuracy and extend the range of validity of our results, we have very recently ${ }^{69}$ expanded our relativistic collision strength program to include an option to use atomic structure data calculated with the well-known multi-configuration DiracFock (MCDF) program of Grant and coworkers, ${ }^{70-72}$ modified by us to handle a large portion of an isoelectronic sequence efficiently. This was used in our recent calculations of the collision strengths for the $45 \Delta n=0$ transitions with $n=2$ in Be-like ions with $8 \leq Z \leq 92$ given in Ref. 73 (preprint attached). It was also used in the similar calculations for the $105 \Delta n=0$ transitions in B-like ions being given in Ref. 74. It does not increase the time of the calculations appreciably because it is the collision part that tends to be the lengthy part. Since the MCDF values for the bound electron contribution to the potential is generally considered to be more accurate than the DFS potential, use of the MCDF potential should provide accurate results for a given isoelectronic sequence for lower $Z$, where the electron-electron contribution to the potential is more significant relative to the contribution of the nucleus. Thus, use of this option probably gives accurate results in most cases for

$$
Z \gtrsim 1.5 N \text { to } 1.8 N
$$

Also the results for high $Z$ should be slightly better. However, test cases done in Ref. 69 indicate very small differences due to use of the MCDF atomic structure data in place of DFS atomic structure data in calculating collision strengths for Be-like ions with $Z$ in the range $8 \leq Z \leq 92$. Thus, perhaps our DFS atomic structure program is valid for lower $Z / N$ values than we had earlier estimated.

In other recent work ${ }^{75,76}$ a large number of test calculations wera made to determine the importance of including the generalized Breit interaction in calculating the scattering matrix elements for excitation of H-like, He-like and Li-like ions with $Z=26,54$ and 92 . For hydrogenic ions the results agree with the early work of Walker ${ }^{77}$ and with recent calculations by Moores and Pindzola. ${ }^{78}$ As expected from the work of Walker on $n=1-2$ excitation of H-like ions, inclusion of the generalized Breit interaction, or equivalently use of the so-called Moller interaction, is not of great importance for $Z \leq 26$ and for higher $Z$ it is principally important only for excitation from the $1 s$ shell. However, we found it to be slightly more important for $n=1-3$ excitation 
than for $n=1-2$ excitation and for very high $Z$ it is not negligible for $n=2-3$ excitation for accuracy at the $10 \%$ level. Also an important and interesting thing found in this work is that the effect of inclusion of the generalized Breit interaction is often greater for excitation from the $1 \mathrm{~s}$ shell in He-like and Li-like ions than in H-like ions and is important for a much larger fraction of transitions. For example, for He-like jons we found a maximum effect of $7 \%$ fur $Z=26$, while the maximum effect for H-like ions with $Z=26$ was only $3.5 \%$. Also for He-like ions with $Z=54$ the near threshold collision strengths for all four transitions involving $1 s-2 p_{1 / 2}$ and $1 s-2 p_{3 / 2}$ were significantly affected by amounts ranging from $-28 \%$ to $+11 \%$, while those for these transitions in H-like ions with $Z=54$ were affected by under $1 \%$. This can be explained as follows: In the more complex He-like and Li-like ions a given orbital transition enters more than one transition. Thus, although the effect on collision strengths summed over $J$ value is similar to that on the corresponding transition in $\mathrm{H}$-like ions, which may be small, the effect can be considerably larger and in opposite directions for the individual transitions in complex ions.

Turning to ionization, we applied the factorization method to ionization in Ref. 79 (reprint attached) and obtained some interesting and important results. In particular we found that when mixing is confined to the states in a complex, that is having the same set of principal quantum numbers, as well as parity and $J$ value, distorted wave ionization cross sections can be expressed in terms of the hydrogen-like cross sections $Q_{H}^{p s}\left(n_{a} l_{a} j_{a}\right)$ mentioned in the previous section regardless of the complexity of the ion. Specifically, $Q_{H}^{p s}\left(n_{a} l_{a} j_{a}\right)$ is exactly like the hydrogenic cross section for ionization from subshell $n_{a} l_{a} j_{a}$ except that the radial functions should be calculated using the appropriate potential for the actual ion being considered. The explicit result is that in general the ionization cross section for the transition from initial ion level $U$ to final ion level $U^{\prime \prime}$ (with one less bound electron) can be written

$$
Q_{\text {ion }}\left(U-U^{\prime \prime}\right)=\sum_{\substack{s, S^{\prime \prime} \\ s_{1}, s_{1}^{\prime \prime}}} B\left(U, S S_{1} ; U^{\prime \prime}, S^{\prime \prime} S_{1}^{\prime \prime}\right) Q_{H}^{p s}\left(n_{a} l_{a} j_{a}\right)
$$

which is similar to, but simpler than, Eq. (1) for excitation. Here $S$ and $S_{1}$ are states contributing to the initial ion level $J$, while $S^{\prime \prime}$ and $S_{1}^{\prime \prime}$ are states ccntributing to the final ion level $U^{\prime \prime}$. The coefficients $B\left(U, S S_{1} ; U^{\prime \prime}, S^{\prime \prime} S_{1}^{\prime \prime}\right)$ depend only on the initial and final ion properties, such as mixing coefficients and angular momentum quantum numbers, and hence are very rapidly calculated. 
It is interesting to note that the very convenient result given by Eq. (4) applies, as well, for photoionization, if photoionization cross sections everywhere replace the corresponding collision cross sections, and in future work we expect to apply it to photoionization.

In applying Eq. (4) to ionization by electron impact in complex cases, where several transitions involve ionization from the same subshell $n_{a} l_{a} j_{a}$, somewhat similar to the discussion of our procedures for excitation, considerable additional computing time is saved if for each impact electron energy the $Q_{H}^{p s}\left(n_{a} l_{a} j_{a}\right)$ are calculated using three values for the transition energy spanning the range of actual transition energies. Then the results for the exact energy of each ionization transition are obtained by Lagrangian interpolation, which is accurate to much better than $1 \%$.

In other work we recently modified the program of Ref. 60 slightly to give cross sections for excitation of highly charged ions to specific magnetic sublevels by directive electrons that are also spin polarized, with spin in the direction of electron propagation. The results were used in Ref. 80 (preprint attached) in determining the degree of polarization of the radiation subsequently emitted by the ions. This should be of interest for some experimental work and possibly for the study of some solar flares. We also considered ionization to specific magnetic sublevels of the final ion due to impact by directive electrons. ${ }^{81}$ This is also of interest for the study of some solar flares, as well as for interyreting EBIT experiments.

It should be mentioned that we have also expanded our programs to give a rapid relativistic autoionization program. Our plans are to apply this in future work to indirect processes such as the resonance contribution to excitation, as we did in Ref. 41 using our earlier more approximate approach. Also we expect to apply it to dielectronic recombination and the innershell excitation autoionization contribution to ionization, which is sometimes the dominant contribution even for high $Z$, as showa in Ref. 82 .

In addition to the work we have described, we have in Ref. 83 (reprint attached) used some of the large amount of relativistic atomic data on collision strengths we have recently calculated to show that the Van Regemorter approximation, ${ }^{84}$ which is still quite widely used for estimating excitation cross sections, is largely very unreliable. Also we have delineated the conditions for which it is an especially poor approximation.

Finally it should be mentioned that the complete set of results for each of our papers on large 
scale calculations of collision strengths and oscillator strengths are on file at the National Energy Research Supercomputer Center at Livermore, CA, under user number 1251. In general the file name for our recent relativistic results for a given isoelectronic sequence is the letter $R$ followed by the abbreviation for the isoelectronic sequence followed in turn by $D A T A$. For example, the file on relativistic results for neon-like ions is named $R N E D A T A$. The files on our earlier results in which relativistic effects were included perturbatively are named similarly, but with the initial letter $R$ omitted.

\section{REFERENCES}

1. D.H. Sampson, Astrophys. J. Suppl. $\underline{28}, 309$ (1974).

2. D.H. Sampson and A.D. Parks, Astrophys. J. Suppl. $\underline{28}, 323$ (1974).

3. A.D. Parks and D.H. Sampson, J. Phys. B: Atom. Molec. Phys. $\underline{8}, 774$ (1975).

4. ___ "Cross Sections and Rates fur the Excitation of Highly Charged Complex Ions by Electron Impact", Penn State, Dept. Astronomy Repr. No. 031 (1976).

5. Astrophys. J. 209, 312 (1976).

6. Phys. Rev. A $\underline{15}, 1382$ (1977).

7. D.H. Sampson, A.D. Parks and R.E.H Clark, Phys. Rev. A $\underline{15}, 1393$ (1977).

8. L.B. Golden and D.H. Sampson, J. Phys. B: Atom. Molec. Phys. 10, 2229 (1977).

9. D.H. Sampson and L.B. Golden, J. Phys. B: Atom. Molec. Phys. 11, 541 (1978).

10. L.B. Golden, D.H. Sampscn and K. Omidvar, J. Phys. B: Atom. Molec. Phys. 11, 3235 (1978).

11. D.H. Sampson, A.D. Parks and R.E.H Clark, Phys. Rev. A 17, 1619 (1978).

12. R.E.H Clark, Comput. Phys. Commun. 16, 119 (1978).

13. L.B. Golden and D.H. Sampson, Astrophys. J. Suppl. $\underline{38}, 19$ (1978).

14. R.E.H. Clark and D.H. Sampson, At. Data Nucl. Data Tables $\underline{22}, 527$ (1978).

15. D.H. Sampson, R.E.H. Clark and A.D. Parks, J. Phys. B: Atom. Molec. Phys. 12, 3257 (1979).

16. D.H. Sampson and L.B. Golden, J. Phys. B: Atom. Molec. Phys. 12, L785 (1979).

17. D.L. Moores, L.B. Golden and D.H. Sampson, J. Phys. B: Atom. Molec. Phys. $\underline{13}, 385$ (1980).

18. L.B. Golden and D.H. Sampson, J. Phys. B: Atom. Molec. Phys. $\underline{13}, 2645$ (1980). 
19. D.H. Sampson and R.E.H. Clark, Astrophys. J. Suppl. $\underline{44}, 169$ (1980).

20. D.H. Sampson, R.E.H. Clark and L.B. Golden, Astrophys. J. Suppl. 44, 193 (1980).

21. R.E.H. Clark, D.H. Sampson and A.D. Parks, Astrophys. J. Suppl. 44, 215 (1980).

22. S.J. Goett, R.E.H. Clark and D.H. Sampson, At. Data Nucl. Data Tables $\underline{25}, 185$ (1980).

23. D.H. Sampson and L.B. Golden, J. Phys. B: Atom. Molec. Phys. 14, 903 (1981).

24. L.B. Golden, R.E.H. Clark, S.J. Goett and D.H. Sampson, Astrophys. J. Suppl. 4ㄷ, 603 (1981).

25. D.H. Sampson, R.E.H. Clark and S.J. Goett, Phys. Rev. A 24, 2979 (1981).

26. R.E.H. Clark, D.H. Sampson and S.J. Goett, Astrophys. J. Suppl. $\underline{49}, 545$ (1982).

27. D.H. Sampson, J. Phys. B: Atom. Molec. Phys. 15, 2087 (1982).

28. S.J. Goett, D.H. Sampson and R.E.H. Clark, At. Data Nucl. Data Tables $\underline{28}, 279$ (1983).

29. D.H. Sampson, S.J. Geett and R.E.H. Clark, At. Data Nucl. Data Tables $\underline{28}, 299$ (1983).

30. ___ At. Data Nucl. Data Tables $\underline{29}, 467$ (1983).

31. S.J. Goett and D.H. Sampson, At. Data Nucl. Data Tables $\underline{29}, 535$ (1983).

32. S.J. Goett, D.H. Sampson and R.E.H. Clark, Astrophys. J. Suppl. 54, 115 (1984).

33. D.H. Sampson, S.J. Goett and R.E.H. Clark, At. Data Nucl. Data Tables $\underline{30}, 125$ (1984).

34. R.E.H. Clark and D.H. Sampson, J. Phys. B: Atom. Molec. Phys. 17, 3311 (198c).

35. D.H. Sampson, S.J. Goett, G.V. Petrou, H.L. Zhang and R.E.H. Clark, At. Data Nucl. Data Tabies $\underline{32}, 343$ (1985).

36. D.H. Sampson, G.V. Petrou, S.J. Goett and R.E.H. Clark, At. Data Nucl. Data Tables $\underline{32}, 403$ (1985).

37. D.H. Sampson, G.M. Weaver, S.J. Goett, H.L. Zhang and R.E.H. Clark, At. Data Nucl. Data Tables $\underline{35}, 223$ (1986).

38. H.L. Zhang, D.H. Sampson and R.E.H. Clark, At. Data Nucl. Data Tables $\underline{35}, 267$ (1986).

39. D.H. Sampson, Phys. Rev. A $\underline{34}, 986$ (1986).

40. H.L. Zhang, D.H. Sampson, R.E.H. Clark and J.B. Mann, At. Data Nucl. Data Tables $\underline{37}, 17$ (1987).

41. H.L. Zhang and D.H. Sampson, Astrophys. J. Suppl. 63, 487 (1987).

42. D.H. Sampson and H. L. Zhang, Phys. Rev. A $\underline{36}, 3590$ (1987).

43. D.H. Sampson and H. L. Zhang, Phys. Rev. A $\underline{37}, 3765$ (1988). 
44. D.H. Sampson, H. L. Zhang and A.K. Mohanty, Phys. Rev. A $\underline{38}, 4569$ (1988).

45. D.H. Sampson and H.L. Zhang, Astrophys. J. $\underline{335}, 516$ (1988).

46. H.L. Zhang, D.H. Sampson, R.E.H. Clark and J.B. Mann, At. Data Nucl. Data Tables $\underline{41}, 1$ (1989).

47. D.H. Sampson, H.L. Zhang, A.K. Mohanty and R.E.H. Clark, Phys. Rev. A $\underline{40}, 604$ (1989).

47. D.H. Sampson, H.L.Zhang, A.K. Mohanty and R.E.H. Clark, “A Dirac-Fock-Slater Approach to Atomic Structure for Highly Charged Ions”, Phys. Rev. A $\underline{40}, 604-615$ (1989).

48. H.L. Zhang, D.H. Sampson and A.K. Mohanty, "Fully Relativistic and Quasirelativistic Distorted Wave Methods for Calculating Collision Strengths for Highly Charged Ions”, Phys. Rev. A $\underline{40}$, 616-632 (1989).

49. H.L. Zhang and D.H. Sampson, "Relativistic Distorted Wave Collision Strengths for Excitation to the $88 n=3$ and $n=4$ Levels in all 71 Neon-Like Ions with $22 \leq Z \leq 92^{n}$, Atom. Data Nucl. Data Tables $\underline{43}, 1-69$ (1989).

50. H.L. Zhang, D.H. Sampson and C.J. Fontes, "Relativistic Distorted Wave Collision Strengths and Oscillator Strengths for the 85 Li-Like lons with $8 \leq Z \leq 92^{n}$, Atom. Data Nucl. Data Tables $\underline{44}, 31-77$ (1990).

51. D.H. Sampson, H.L. Zhang and C.J. Fontes, "Relativistic Distorted Wave Collision Strengths and Oscillator Strengths for the 71 Na-Like Ions with $22 \leq Z \leq 92$ ", Atom. Data Nucl. Data Tables $\underline{44}$, 209-271 (1990).

52. H.L. Zhang, D.H. Sampson and C.J. Fontes, "Relativistic Distorted Wave Collision Strengths and Oscillator Strengths for the $33 \mathrm{Cu}$-Like Ions with $60 \leq Z \leq 92$ ", Atom. Data Nucl. Data Tables $\underline{44}$, 273-304 (1990).

53. A. Bar-Shalom, M. Klapisch and J. Oreg, Phys. Rev. A $\underline{38}, 1773$ (1988).

54. H.L. Zhang and D.H. Sampson, "A Rapid Relativistic Distorted Wave Approach for Calculating Cross Sections for Ionization of Highly Charged Ions”, Phys. Rev. A $\underline{42}, 5378-5383$ (1990).

55. M. S. Pindzola and M. J. Buie, Phys. Rev. A $\underline{37}, 3232$ (1988).

56. M. S. Pindzola and M. J. Buie, Phys. Rev. A $\underline{39}, 1029$ (1989).

57. M. S. Pindzola, D. L. Moores and D. C. Griffin, Phys. Rev. A $\underline{40}, 4941$ (1989).

58. D. L. Moores and M. S. Pindzola, Phys. Rev. A $\underline{41}, 3603$ (1990). 
59. D. L. Moores and M. S. Pindzola, Phys. Rev. A $\underline{42}, 5384$ (1990).

60. H.L. Zhang, D.H. Sampson and R.E.H. Clark, "Relativistic Cross Sections for Excitation of Highly Charged Ions to Specific Magnetic Sublevels by an Electron Beam”, Phys. Rev. A 41 , 198-206 (1990).

61. R. E. Marrs, M. A. Levine, D. A. Knapp and J. R. Henderson, Phys. Rev. Lett. 60 , 1715 (1988).

62. P. Beiersdorfer, A.L. Osterheld, M.H. Chen, J.R. Henderson, D.A. Knapp, M.A. Levine, R.E. Marrs, K.J. Reed, M.B. Schneider, and D.A. Vogel, Phys. Rev. Lett. $\underline{65}$, 1995 (1991).

63. P. Beiersdorfer, M.H. Chen, R.E. Marrs, M.B. Schneider, and R.S. Walling, Phys. Rev. A $\underline{44}$, 396 (1991).

64. P. Beiersdorfer, private communication.

65. C. Bhalla, private communication.

66. D.H. Sampson, H.L. Zhang and C.J. Fontes, "Relativistic Distorted Wave Collision Strengths and Oscillator Strengths for F-Like Ions with $22 \leq Z \leq 92^{n}$, Atom. Data Nucl. Data Tables $\underline{48}$, 25-90 (1991).

67. M.H. Chen, B. Crasemann, M. Aoyagi, K.-N. Huang and H. Mark, At. Data Nucl. Data Tables $\underline{26}, 561(1981)$.

68. H.L. Zhang, D.H. Sampson and C.J. Fontes, "Relativistic Distorted Wave Collision Strengths and Oscillator Strengths for the $33 \mathrm{Ni}$-Like Ions with $60 \leq Z \leq 92$ ", Atom. Data Nucl. Data Tables $\underline{48}, 91-163$ (1991).

69. H.L. Zhang and D.H. Sampson, "Tests of and Improvements in a Fully Relativistic Distorted Wave Method for Calculating Collision Strengths for Highly Charged Ions", being prepared for submission to Phys. Rev. A.

70. I. P. Grant, B. J. McKenzie, P. H. Norrington, D. F. Mayers, and N. C. Pyper, Comput. Phys. Commun. 21, 207 (1980).

71. B. J. McKenzie, I. P. Grant and P. H. Norrington, Comput. Phys. Commun. 21,233 (1980).

72. K.G. Dyall, I.P. Grant, C.T. Johnson, F.A. Parpia and E.P. Plummer, Comput. Phys. Commun. $\underline{55}, 425$ (1989).

73. H.L. Zhang and D.H. Sampson, "Relativistic Distorted Wave Collision Strengths and Oscillator Strengths for the $45 \Delta n=0$ Transitions with $n=2$ in the 85 Be-Like Ions with $8 \leq Z \leq 92$ ", 
submitted to Atom. Data Nucl. Data Tables.

74. H.L. Zhang and D.H. Sampson, "Relativistic Distorted Wave Collision Strengths and Oscillator Strengths for the $105 \Delta n=0$ Transitions with $n=2$ in the 85 B. Like Ions with $8 \leq Z \leq 92$ ", being prepared for submission to Atom. Vata Nucl. Data Tables.

75. C.J. Fontes, Ph. D. Thesis, The Pennsylvania State University.

76. C.J. Fontes, D.H. Sampson and H.L. Zhang, "Inclusion of the Generalized Breit Interaction in Calculating the Matrix Elements for Excitation of Highly Charged Ions", being prepared for submission to Phys. Rev. A.

77. D. W. Walker, J. Phys. B $\underline{8}, 760$ (1975).

78. D. L. Moores and M. S. Pindzola, private communication and being submitted to J. Phys. B (1992).

79. D.H. Sampson and H.L. Zhang, "Rapid Relativistic Ionization Approach Based on the Factorization Method", Phys. Rev. A $\underline{45}$, 1657-1669 (1992).

80. M.K. Inal, H.L. Zhang and D.H. Sampson, "Circular Polarization of the FeXXV X-ray Lines Following Collisional Excitation by Longitudinally Polarized Electrons", accepted by Phys. Rev. A.

81. H.L. Zhang, D.H. Sampson and M.K. Inal, "Relativistic Cross Sections for Ionization of Highly Charged Ions to Specific Magnetic Substates by an Electron Beam", being prepared for submission to Phys. Rev. A.

82. K. J. Reed, M. H. Chen and D. L. Moores, Phys. Rev. A $\underline{41}, 550$ (1990).

83. D.H. Sampson and H.L. Zhang, "On Use of the Van Regemorter Formula for Collision Strengths or Cross Sections", Phys. Rev. A $\underline{45}, 1556-1561$ (1992).

84. H. Van Regemorter, Astrophys. J $\underline{136}, 906$ (1962). 
DATE FILMED 12128192 


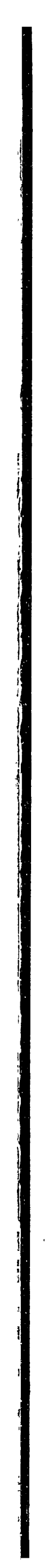

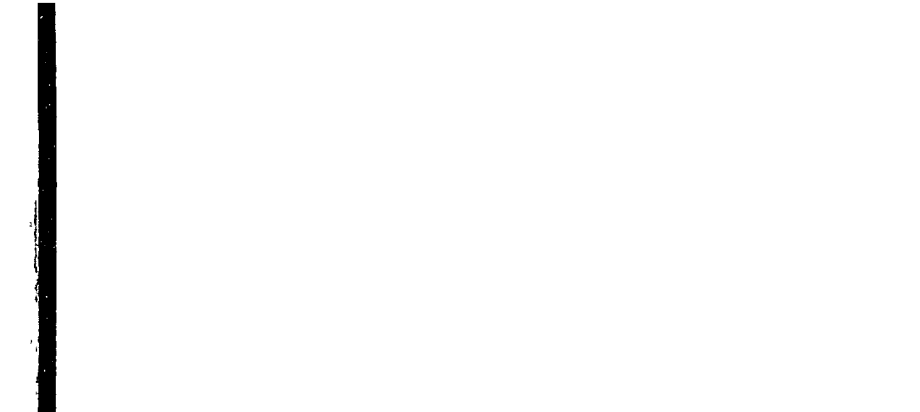

